leading to robust transcriptional activation (Fig.1). This proposed mechanism bears a resemblance to the process of forming an immense snowball through aggregation by rolling it down a snow-clad hillside. However, this 'snowball effect' directed by chrRBPs may cause unwanted consequences because any fundamental cellular process, such as gene transcription, requires both initiation and termination mechanisms for sophisticated regulation. Indeed, it has been shown that the transcriptional condensates in cells are highly dynamic, rapidly forming and dissolving over a timescale of several minutes ${ }^{9}$. Notably, RNA seems able to either promote or impede phase separation, presumably depending on the charge balance of RNA and protein present in the local environment ${ }^{10}$. With this in mind, it will be important to further dissect the roles of RBPs at the resolving phase of transcriptional condensates. For example, do RBPs with distinct phase-separation properties have different impacts on the fate of transcriptional condensates? Could post-translational modification of RBPs provide an additional layer of regulation in this process? Addressing these questions will likely offer a more comprehensive view of the functions of RBPs in transcriptional regulation.

Furthermore, how the specificity of transcription regulation by RBPs is established remains an outstanding question. On the basis of the authors' model, nascent RNA seems to recruit RBPs to wrap around the transcribing sites in a sequence-independent manner. Nevertheless, many RNAs can associate with chromatin through trans interactions, which likely enlist specific RBPs to execute their functions on site ${ }^{6}$. Could the trans-acting RNAs provide RBPs with the specificity that controls where they reside? Alternatively, many DNA-bound factors have been shown to interact with RBPs, potentially offering a different means to guide RBPs onto chromatin. Thus, the specificity of RBPs that act on transcription is likely to be context dependent and awaits further investigation.

In summary, the discovery of RBPs' role in regulating transcription through phase-separated condensates provides new insights into how the RNA-directed and chromatin-bound RBPs control gene expression at the transcriptional level. In light of the ever-expanding list of RBPs identified, future studies will be needed to continue to push the edges of what we know about the functionality of RBPs in gene expression control.

\section{Chengyu Li and Huasong Lu(D) $\bowtie$ Zhejiang Provincial Key Laboratory for Cancer Molecular Cell Biology, Life Sciences Institute, Zhejiang University, Hangzhou, Zhejiang, China.

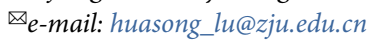

Published online: 16 December 2021 https://doi.org/10.1038/s41589-021-00902-7

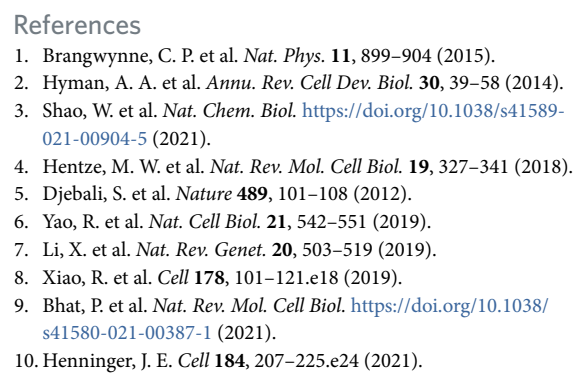

Competing interests

The authors declare no competing interests.

\title{
Host glycolipids in SARS-CoV-2 entry
}

\author{
A new study demonstrates that the receptor-binding domain of the SARS-CoV-2 spike protein binds to sialylated \\ glycans, especially glycolipids, to facilitate viral entry, an insight that identifies new potential targets for \\ SARS-CoV-2 interventions.
}

\section{Ryuta Uraki and Yoshihiro Kawaoka}

S evere acute respiratory syndrome coronavirus 2 (SARS-CoV-2), which is responsible for the ongoing global COVID-19 pandemic, initiates infection of human cells by binding to angiotensin-converting enzyme 2 (ACE2) via the receptor-binding domain (RBD) of its spike protein ${ }^{1}$. Cellular transmembrane serine protease 2 (TMPRSS2) and neuropilin-1 are key host factors for the virus infection ${ }^{2-4}$. In addition to these host proteins, the function of cellular glycans during SARS-CoV-2 infection is of interest, and Nguyen and colleagues ${ }^{5}$ now identify specific glycans that interact with SARS-CoV-2 RBD using a highly sensitive screen of glycan libraries to unlock the roles of host glycans in SARS-CoV-2 infection.
Because the interactions between host cell-surface factors and viruses define steps in the viral life cycle, including virus attachment and internalization, unraveling the complicated interactions between host cell-surface factors and viruses is essential to understand the viral entry step. Although several proteins, such as ACE2 and TMPRSS2, have been well documented as important host factors for SARS-CoV-2 infection, ACE2-independent infections have been reported, which suggests that our current knowledge of the viral entry process for SARS-CoV-2 is still limited and further investigation is needed ${ }^{6}$. Beyond proteins, glycans have been known to have important roles in the life cycles of many viruses, such as orthomyxoviruses, flaviviruses, reoviruses and adenoviruses. Several groups have recently suggested that heparan sulfate may serve as a co-receptor for SARS-CoV-2, which raises interest in the potential role of host glycans during SARS-CoV-2 infection ${ }^{7}$.

Klassen and colleagues ${ }^{8}$ previously developed a catch-and-release electrospray ionization mass spectrometry (CaR-ESI-MS) assay that identifies carbohydrate-protein interactions with low affinity. In the current study ${ }^{5}$, this technique is applied for screening carbohydrate libraries against the SARS-CoV-2 RBD and the full spike protein. The authors reveal that both the RBD and spike proteins can bind to various acidic glycans, with especially high preference for the oligosaccharides on the monosialic gangliosides GM1 and GM2, which belong 
a

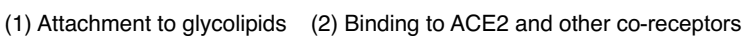

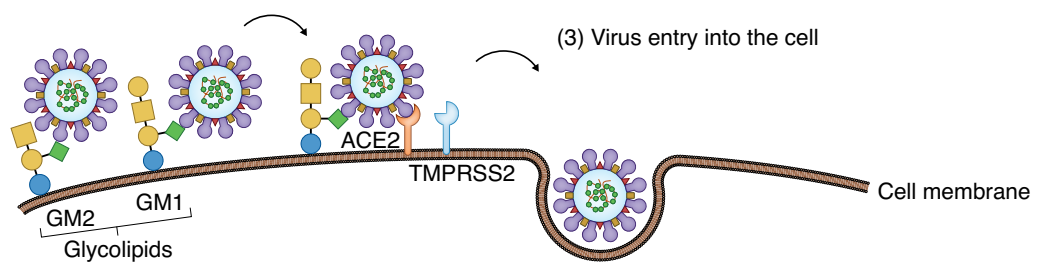

Galactose $\square N$-acetylgalactosamine $\diamond N$-acetylneuraminic acid (sialic acid) $\bigcirc$ Glucose $\bigcirc \mathrm{N} \Delta \mathrm{E} \square \mathrm{M} \odot \mathrm{S}$

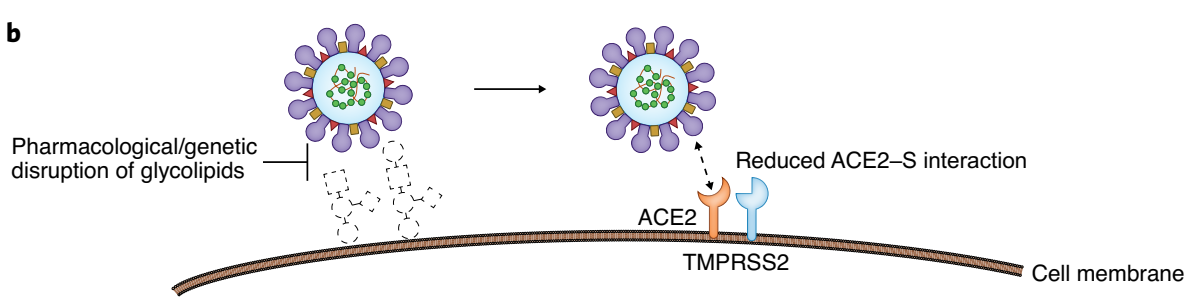

Fig. 1 A role for glycolipids in SARS-CoV-2 infection. a, As an initial step of SARS-CoV-2 entry, the RBD of SARS-CoV-2 spike (S) protein binds to sialylated glycans, specifically glycolipids such as GM1 or GM2. The interaction between the RBD and the sialylated glycans drives the recognition of ACE2 by the spike protein, which facilitate the infection. b. Several strategies such as pharmacological inhibitors or genetic disruption of glycolipid synthesis lead to reduced SARS-CoV-2 infection, which could be a strategy to control SARS-CoV-2 infection.

to a class of glycolipids that are found predominantly in the outer leaflet of the plasma membrane (Fig. 1a). Although other coronaviruses, such as bovine $\mathrm{CoV}$ and $\mathrm{HCoV}-\mathrm{OC} 43$, are known to bind glycolipids through the $\mathrm{N}$-terminal domain of their spike protein ${ }^{9}$, it is noteworthy that the RBD of SARS-CoV-2 has a strong binding affinity for glycolipids, such as GM1 and GM2.

Nguyen et al..$^{5}$ next examined the importance of sialic acid for RBD binding to cells by using pharmacological, genetic or enzymatic abrogation of sialic acid in vitro and show that reduction of sialic acid on cells causes decreased pseudotyped viral infectivity, which suggests that SARS-CoV-2 entry via its RBD is dependent on sialic acid $^{3}$. It should be noted, however, that the contribution of sialic acid to SARS-CoV-2 entry is smaller than that of ACE2.

Given that glycolipids such as GM1 and GM2 are top hits in the CaR-ESI-MS assay, the authors assessed whether the infection of pseudovirus or authentic SARS-CoV-2 depends on glycolipids. Pharmacological or genetic abrogation of a key enzyme in glycolipid biosynthesis, UDP-glucose ceramide glycosyltransferase, decreased
RBD binding and SARS-CoV-2 infection, which suggests a key role for glycolipids in SARS-CoV-2 entry (Fig. 1b).

This study raises several questions for future investigation. How do sialylated glycans support virus entry? Do they affect the levels of expression and localization of viral receptors, such as ACE2 and TMPRSS2? A detailed structural analysis of the interaction between ACE2 and glycolipids would be highly desirable. As host sialylated glycans are known to regulate the innate and adaptive immune responses ${ }^{10}$, does the pathogen-glycan interaction alter not only the entry of SARS-CoV-2 but also the induced immune responses? This study uses the prototype SARS-CoV-2 and D614G strains to assess the importance of sialylated glycans including glycolipids for virus entry. At present, many variants with amino acid mutations in the spike protein including the $\mathrm{RBD}$ are emerging and circulating in the world. Among these variants, variants of concerns possess an increased risk to global public health owing to altered antigenicity, enhanced transmissibility or pathogenicity. Therefore, another important question is whether amino acid substitutions in the spike protein of variants of concerns affect the affinity between the spike protein and host sialylated glycans, and thereby viral infectivity. Future studies that build on the findings of Nguyen et al. ${ }^{5}$ will help to further determine the importance of this previously unrecognized involvement of host glycolipids in SARS-CoV-2 infection.

Although developing new drugs against SARS-CoV-2 is highly desirable, it is challenging, and no antiviral compounds that specifically target this virus have yet been approved by the US Food and Drug Administration. The discovery of sialylated glycans, especially glycolipids, as a key host factor for viral infection provides an important insight into the virus life cycle and unlocks the potential for new antiviral treatments. Beyond SARS-CoV-2, the glycan library screening strategy using the CaR-ESI-MS assay could be applicable not only to future emerging viruses but also to other pathogens such as bacteria and parasites, which could also lead to development of drugs against those pathogens.

Ryuta Uraki (D) 1,2 and Yoshihiro Kawaoka (D) 1,2,3,4凶

${ }^{1}$ The Research Center for Global Viral Diseases, National Center for Global Health and Medicine Research Institute, Tokyo, Japan. ${ }^{2}$ Division of Virology, The Institute of Medical Science, The University of Tokyo, Tokyo, Japan. ${ }^{3}$ Department of Special Pathogens, International Research Center for Infectious Diseases, The Institute of Medical Science, The University of Tokyo, Tokyo, Japan. ${ }^{4}$ Department of Pathobiological Sciences, School of Veterinary Medicine, University of Wisconsin-Madison,

Madison, WI, USA.

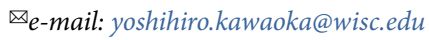

Published online: 9 November 2021 https://doi.org/10.1038/s41589-021-00923-2

References

1. Hoffmann, M. et al. Cell 181, 271-280 (2020).

2. Cantuti-Castelvetri, L. et al. Science 370, 856-860 (2020).

3. Daly, J. L. et al. Science 370, 861-865 (2020).

4. Bestle, D. et al. Life Sci. Alliance 3, e202000786 (2020).

5. Nguyen, L. et al. Nat. Chem. Biol. https://doi.org/10.1038/s41589021-00924-1 (2021).

6. Puray-Chavez, M. et al. Cell Rep. 36, 109364 (2021).

7. Clausen, T. M. et al. Cell 183, 1043-1057 e1015 (2020).

8. Zhang, Y. et al. Anal. Chem. 84, 7618-7621 (2012).

9. Tortorici, M. A. et al. Nat. Struct. Mol. Biol. 26, 481-489 (2019).

10. van Kooyk, Y. \& Rabinovich, G. A. Nat. Immunol. 9 , 593-601 (2008).

Competing interests

The authors declare no competing financial interests. 